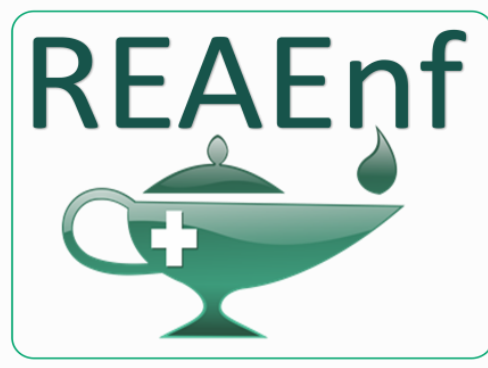

Revista Eletrônica Acervo Enfermagem
REVISÃO BIBLIOGRÁFICA

Recebido em: 11/2019

Aceito em: $12 / 2019$

Publicado em: 2/2020

\title{
O impacto da doença de Alzheimer na vida do cuidador
}

\author{
The impact of Alzheimer's disease on caregiver life
}

\section{El impacto de la enfermedad de Alzheimer en la vida del cuidador}

Priscila Dutra Miranda Silva ${ }^{1 *}$, Jonathan Mendes de Castro ${ }^{1}$, Ranyelli Estefany da Costa Martins ${ }^{1}$, Paloma Calazans Galinari ${ }^{1}$, Marcela Alves Azevedo ${ }^{1}$, Talles Vinícius de Castro Oliveira ${ }^{1}$, Enaile de Souza Proti ${ }^{1}$, Ariane Candido ${ }^{1}$, Carlos Henrique Wernersbach Guerra ${ }^{1}$, Wendel Jose Teixeira Costa $^{1,2}$.

Resumo: Essa pesquisa objetivou reunir e sistematizar resultados de investigações científicas relacionadas aos impactos sobre a saúde sofridos pelos cuidadores de pessoas com Doença de Alzheimer (DA). Trata-se de uma revisão integrativa da literatura sobre os impactos da DA sobre à saúde dos cuidadores. A coleta de dados ocorreu em julho de 2019 e foram utilizadas as bases de dados eletrônicos Literatura Latino-Americana e do Caribe em Ciências da Saúde (LILACS) e US National Librare of Medicine (PubMed), com os descritores doença de alzheimer e adaptação familiar, no idioma ingles, combinados com o operador booleano (AND). A amostra final foi constituída por nove artigos, sendo sete artigos da PubMed e dois da LILACS. Observou-se alta sobrecarga de estresse e difilculdades de enfrentamento observados nos cuidadores de familiares com DA. Declínio na vida social, no lazer, no auto-cuidado, no tempo dedicado a sua vida particular, geram impactos negativos, e com passar do tempo traz problemas à sua saúde, sendo imprescindível uma atenção voltada ao cuidador, a sua saúde, a fim de evitar o desenvolvimento e o surgimento de doenças que podem afetar sua vida e trazer prejuízos que podem ser até mesmo irreverssíveis.

Palavras-chave: Doença de Alzheimer, Adaptação, Cuidador familiar.

Abstract: This research aims to gather and systematize results of scientific research related to health impacts suffered by caregivers of people with Alzheimer's (AD). This is an integrative literature review on the impacts of $A D$ on the health of caregivers. Data collection took place in July 2019 and was used as an electronic database Latin American Literature in Health Sciences (LILACS) and US National Librare of Medicine (PubMed), with the descriptors of alzheimer disease and family adaptation, without English language, combined with the Boolean operator (AND). A final sample was selected by nine articles, seven articles from PubMed and two from LILACS. High stress overload and coping difficulties were observed in caregivers of relatives with $A D$. Decline in social life, no leisure, no self-care, no time devoted to your private life, negative effects are generated, and passing time brings problems to your health, requiring attention to the caregiver, their health, an end to prevent the development and treatment of diseases that can affect your life and cause damage that may even be irreversible.

Keywords: Alzheimer disease, Adaptation, Caregiver.

\footnotetext{
${ }^{1}$ Centro Universitário de Caratinga (UNEC), Caratinga - MG.

*E-mail: prisciladmiranda@gmail.com

${ }^{2}$ Laboratório de Escrita Científica, Escola Superior de Ciências da Santa Casa de Misericórdia (EMESCAM), Vitória - ES.
} 
Resumen: Esta investigación tuvo como objetivo recopilar y sistematizar los resultados de investigaciones científicas relacionadas con los impactos en la salud que sufren los cuidadores de personas con enfermedad de Alzheimer (EA). Esta es una revisión integradora de la literatura sobre los impactos de la EA en la salud de los cuidadores. La recolección de datos tuvo lugar en julio de 2019 y se utilizaron las bases de datos electrónicas Literatura Latino-Americana em Ciências da Saúde (LILACS) e US National Librare of Medicine (PubMed), con los descriptores de enfermedad de Alzheimer y adaptación familiar, en inglés, combinados con el operador booleano (AND). La muestra final consistió en nueve artículos, siete artículos de PubMed y dos de LILACS. Se observó una alta sobrecarga de estrés y dificultades de afrontamiento en los cuidadores de familiares con EA. La disminución de la vida social, el ocio, el autocuidado, el tiempo dedicado a su vida privada, generan impactos negativos y, con el tiempo, traen problemas a su salud, es una atención esencial centrada en el cuidador, su salud, para para prevenir el desarrollo y la aparición de enfermedades que pueden afectar su vida y causar daños que incluso pueden ser irreversibles.

Palabras clave: Enfermedad de Alzheimer, Adaptación, Cuidador familiar.

\section{INTRODUÇÃO}

A demência é o comprometimento cognitivo progressivo adquirido suficiente para impactar nas atividades da vida diária configurando-se com uma das principais causas de dependência, incapacidade e mortalidade. As estimativas sugerem que atualmente 44 milhões de pessoas vivem com demência em todo o mundo. Prevê-se que esse número triplique em 2050 à medida que a população envelhece (SILVA MVF, 2019).

A Doença de Alzheimer (DA) é a maior causa isolada de demência, representando $50 \%$ a $75 \%$ dos casos, com maior prevalência entre idosos, quase dobrando a cada 5 anos após os 65 anos. Caracteriza-se por disfunção cognitiva e neurodegeneração progressiva. Geralmente se manifesta através da perda progressiva de memória episódica e da função cognitiva, causando deficiências de linguagem e habilidades visuoespaciais, que são frequentemente acompanhadas por distúrbios comportamentais como apatia, agressividade e depressão (JING W, et al., 2016; SILVA MVF, 2019).

A patogênese da DA é multifatorial, envolvendo lesão vascular e morte neuronal não isquêmica, levando a graves conseqüências para a cognição, maior do que qualquer patologia isolada (BORSHCHEV YY, et al., 2019). Aproximadamente $70 \%$ do risco de desenvolver DA pode estar atribuído à genética. No entanto, fatores adquiridos como doenças cerebrovasculares, diabetes, hipertensão, obesidade e dislipidemia aumentam o risco de desenvolvimento de doença (SILVA MVF, 2019).

A DA é a principal causa de demência e incapacitação nos idosos, gerando altos custos de saúde. No Brasil, a prevalência estimada de pessoas com demências foi de 390.000 casos no ano 2000, sendo a DA responsável por mais da metade dos casos (WORLD HEALTH ORGANIZATION, 2012).

A pessoa com DA perde progressivamente a memória e a autonomia, tornando-se dependente de outros para a execução de tarefas simples e do autocuidado (JING W, et al., 2016; SILVA MVF, 2019). Por se tratar de uma doença crônica debilitante, a maior parte das pessoas com DA são cuidadas em casa, o que acarreta em seus cuidadores altos níveis de sobrecarga e estresse (CZEKANSKI K, 2017). Diante disso, cuidar de um paciente com DA representa um desafio real que pode ter consequências psicológicas e físicas consideráveis em longo prazo, para o cuidador (MONTEIRO AMF, et al., 2018).

A função de cuidar de um membro da família com demência pode deixar pouco tempo para os cuidadores cuidarem de suas próprias necessidades de saúde, o que os torna mais suscetíveis a problemas de saúde mental e física (PARKER G, et al., 2010).

Cuidadores de pessoas com DA relatam estresse, sobrecarga e depressão significativos, em comparação com cuidadores de pessoas com outras demências, especialmente quando os sintomas neuropsiquiátricos são proeminentes. Estratégias de enfrentamento adequadas podem modificar o impacto de situações estressantes e aumentar a qualidade de vida dos cuidadores (CHEN CT, et al., 2017).

Quando o cuidador é um membro da família, a sobrecarga e o estresse podem ser ainda maiores, gerando consequências negativas para a saúde física e mental dos cuidadores, devido aos altos níveis de carga, 
estresse e solidão, frequentemente resultantes dessa função (MOON H e DILWORTH-ANDERSON P, 2015; LUCHESI BM, et al., 2015).

Diante desse contexto, o presente estudo busca reunir e sistematizar resultados de investigações científicas relacionadas aos impactos, sobre a saúde, sofridos pelos cuidadores de pessoas com DA.

\section{MÉTODOS}

Trata-se de uma revisão integrativa da literatura sobre os impactos, à saúde, sofridos pelo cuidador de pessoas com DA. Para o desenvolvimento da presente revisão foram percorridas as seguintes etapas: elaboração da questão norteadora, busca na literatura, seleção dos artigos e coleta de dados, análise crítica dos estudos incluídos, discussão dos resultados e apresentação da revisão integrativa.

A pesquisa partiu da seguinte questão norteadora: Quais são os impactos, sobre a saúde, sofridos pelos cuidadores de pessoas com DA?

A coleta de dados ocorreu durante setembro de 2019 e foram utilizadas na seleção dos artigos, as seguintes bases de dados eletrônicos: Literatura Latino-americana e do Caribe em Ciências da Saúde (LILACS) e US National Librare of Medicine (PubMed).

Empregaram-se os Descritores em Ciências da Saúde (DeCS): Doença de Alzheimer e Adaptação Familiar, no idioma inglês (Alzheimer Disease, Family Adaptation), combinados com o operador booleano (AND), com recorte temporal dos últimos 5 anos, com disponibilidade de texto completo. Foi possível a captura de 189 artigos. Na Tabela 1, encontra-se o roteiro de busca utilizado para a composição da amostra.

Tabela 1 - Distribuição dos estudos capturados segundo combinação dos descritores.

\begin{tabular}{lccc}
\hline \multicolumn{2}{c}{ Descritores Combinados } & $\begin{array}{c}\text { Estudos encontrados na } \\
\text { PubMed }\end{array}$ & $\begin{array}{c}\text { Estudos encontrados em } \\
\text { LILACS }\end{array}$ \\
\hline $\begin{array}{l}\text { Alzheimer Disease } \\
\text { Family Adaptation }\end{array}$ & AND & 57 & 13 \\
\hline
\end{tabular}

Fonte: Silva PDM, et al., 2019.

Foram incluídos artigos disponíveis na íntegra e que respondessem à questão norteadora da pesquisa. Foram excluídas, teses, dissertações e publicações que não respondessem à questão norteadora.

O processo de análise e síntese dos artigos foi embasado no instrumento elaborado e validado por Ursi ES e Gavão CM (2006), e realizado de forma descritiva, possibilitando observar, contar, descrever e classificar os dados, com o intuito de reunir o conhecimento produzido sobre o tema explorado na revisão, sendo apreendidas as seguintes informações: autoria dos artigos, ano de publicação, objetivos, temática, resultado e considerações relevantes.

Para a avaliação e apresentação dos artigos incluídos na revisão, foi construído um quadro comparativo descrevendo as principais informações e características dos artigos.

\section{RESULTADOS}

A amostra final foi constituída por nove artigos, sendo sete artigos da PubMed e dois da LILACS, com média de 2 estudo por ano, considerando o período de publicação dos artigos incluídos. As temáticas centrais foram os impactos e mudanças que os cuidadores sofrem cuidando de pacientes com Alzheimer e formas de enfrentamento. Os métodos de pesquisa foram predominantemente observacionais, descritivos e qualitativos.

Quanto à autoria dos estudos, a maior parte dos pesquisadores identificados são pesquisadores ligados a programas de pós-graduação e docentes. 
Tabela 2 - Artigos levantados nas bases de dados PubMed e LILACS.

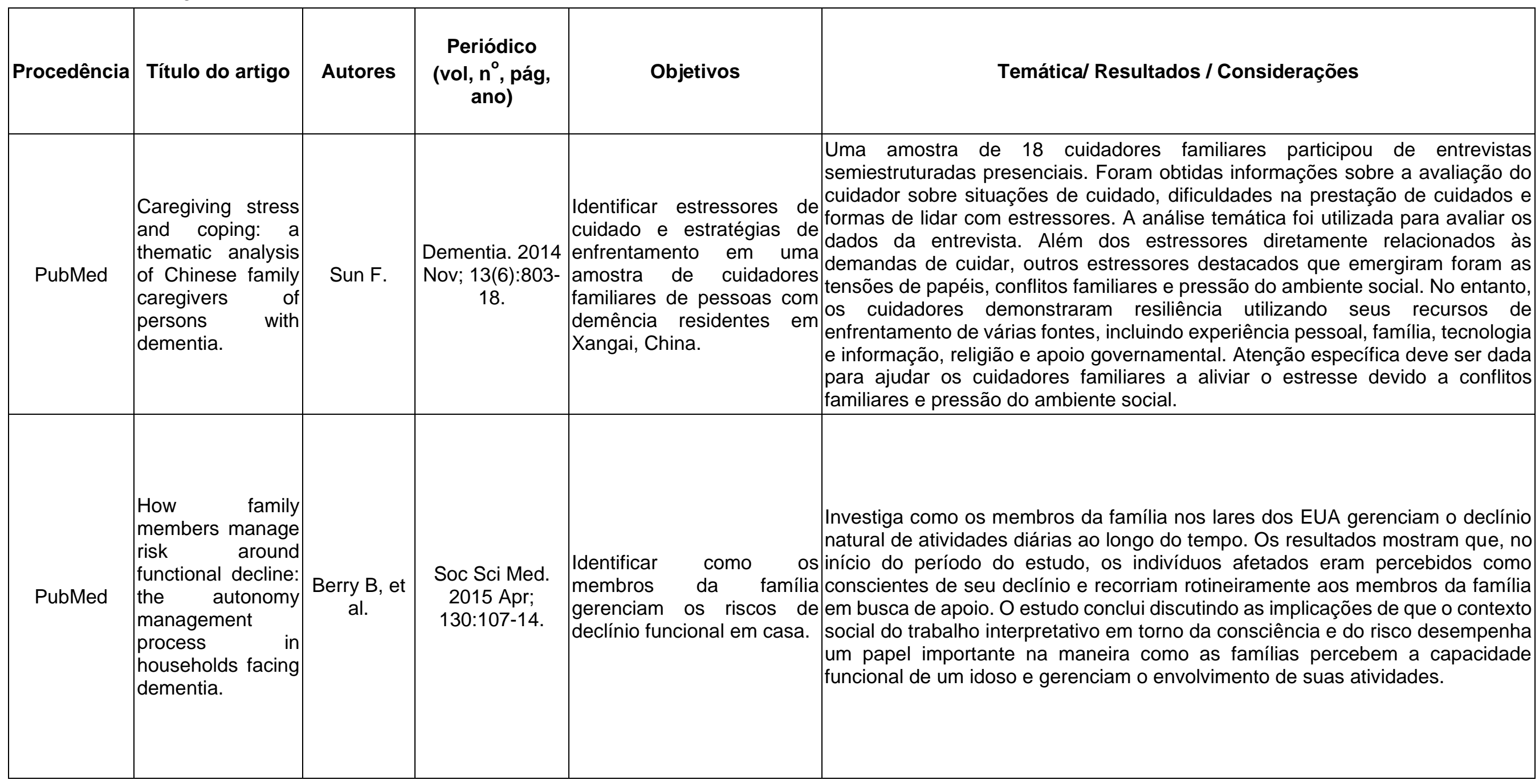

REAEnf/EJNC | Vol. 2 | e2353 | DOI: https://doi.org/10.25248/REAenf.e2353.2020 Página 4 de 10 


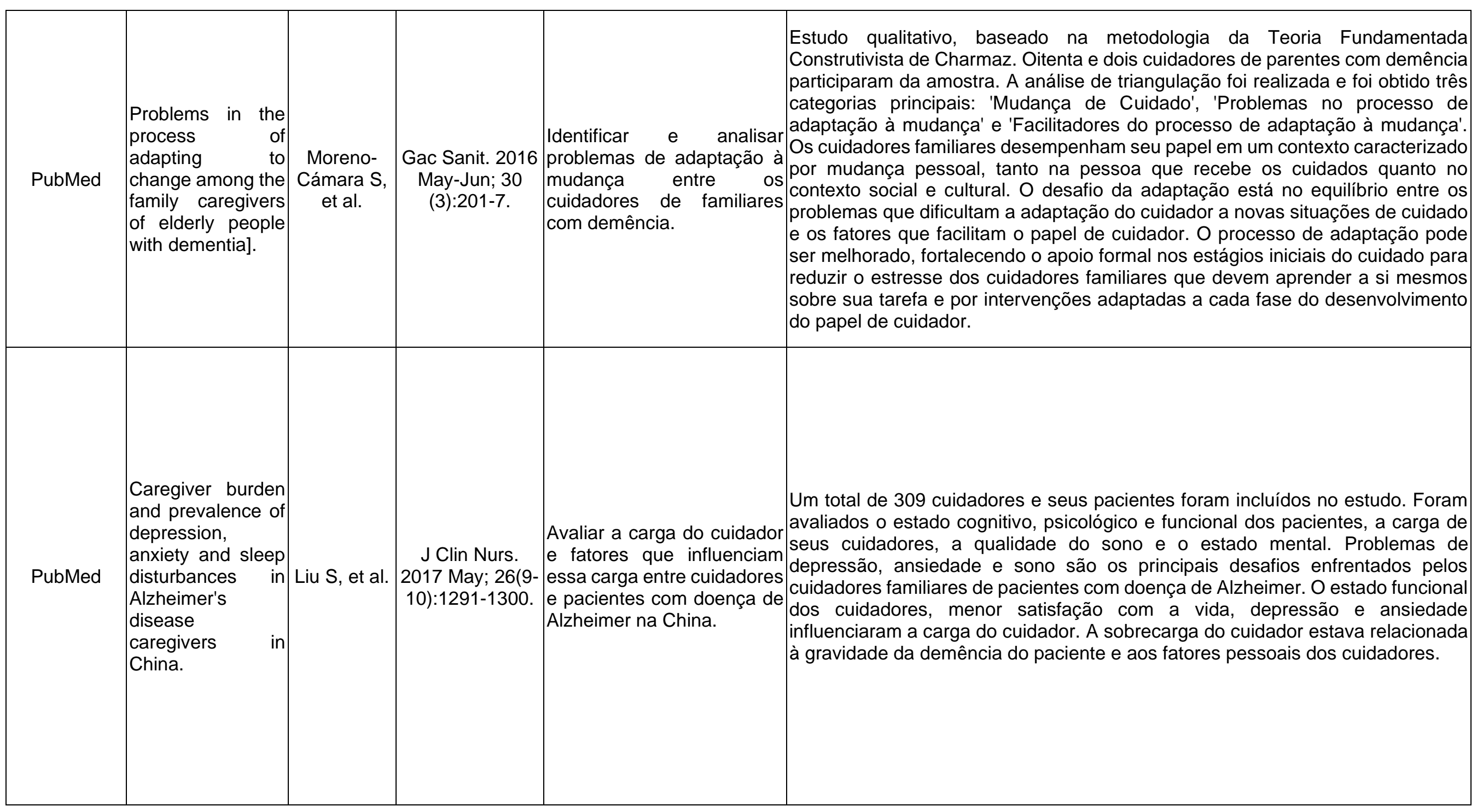

REAEnf/EJNC | Vol. 2 | e2353 | DOI: https://doi.org/10.25248/REAenf.e2353.2020 Página 5 de 10 


\begin{tabular}{|c|c|c|c|c|c|}
\hline PubMed & $\begin{array}{l}\text { The relationship } \\
\text { between } \\
\text { caregiving self- } \\
\text { efficacy } \\
\text { depressive and } \\
\text { symptoms in } \\
\text { family caregivers } \\
\text { of patients with } \\
\text { Alzheimer } \\
\text { disease: } \\
\text { longitudinal study. }\end{array}$ & $\begin{array}{c}\text { Grano C, et } \\
\text { al. }\end{array}$ & \begin{tabular}{|} 
IntPsychogeriatr. \\
2017 Jul; \\
29(7):1095- \\
1103.
\end{tabular} & 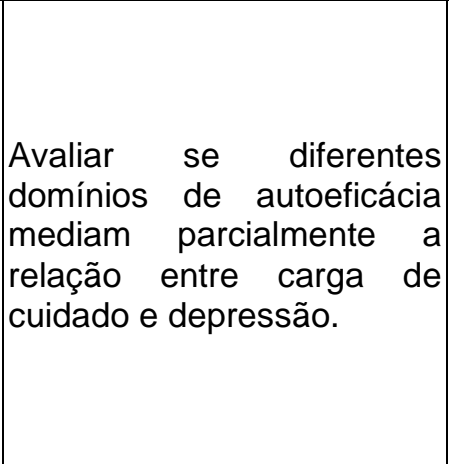 & $\begin{array}{l}\text { Cento e setenta cuidadores de pacientes com DA responderam a medidas de } \\
\text { carga do cuidador, autoeficácia do cuidador e sintomas depressivos. Os dados } \\
\text { foram analisados por meio de modelos de equações estruturais. A carga no da } \\
\text { primeira avaliação influenciou significativamente a depressão um ano depois e a } \\
\text { relação entre a carga no momento um e os sintomas depressivos um ano depois } \\
\text { foi parcialmente mediada pela autoeficácia no controle de pensamentos } \\
\text { perturbadores. Os achados do presente estudo fornecem evidências de que, } \\
\text { durante um período considerável de tempo, os efeitos da carga do cuidador sobre } \\
\text { os sintomas depressivos podem ser explicados pelas crenças de eficácia dos } \\
\text { cuidadores no controle de pensamentos perturbadores relacionados às tarefas } \\
\text { de cuidar. }\end{array}$ \\
\hline PubMed & $\begin{array}{l}\text { Neuropsychiatric } \\
\text { symptoms in } \\
\text { Alzheimer's } \\
\text { disease: } \\
\text { associations with } \\
\text { caregiver burden } \\
\text { and treatment } \\
\text { outcomes. }\end{array}$ & $\begin{array}{l}\text { Chen CT, } \\
\text { et al. }\end{array}$ & \begin{tabular}{|c} 
QJM. 2017 Sep \\
$1 ; 110(9): 565-$ \\
570.
\end{tabular} & $\begin{array}{l}\text { Abordar o impacto dos } \\
\text { sintomas neuropsiquiátricos } \\
\text { de pacientes em diferentes } \\
\text { estágios da DA na carga de } \\
\text { seus cuidadores. }\end{array}$ & $\begin{array}{l}\text { Todos os pacientes foram testados usando o mini exame do estado mental } \\
\text { (MEEM), o instrumento de triagem de habilidades cognitivas (CASI), o inventário } \\
\text { neuropsiquiátrico (NPI) e a escala CDR. Dados sobre os resultados terapêuticos } \\
\text { dos medicamentos também foram coletados. Os cuidadores foram testados } \\
\text { usando o NPI. A gravidade dos sintomas neuropsiquiátricos em pacientes com } \\
\text { DA foi positivamente associada ao estresse do cuidador, e pacientes com } \\
\text { melhores funções cognitivas, em tratamento com medicamentos tiveram } \\
\text { melhores resultados terapêuticos. Para reduzir o impacto dos sintomas } \\
\text { neuropsiquiátricos, é crucial detectar demência em suas fases iniciais e fornecer } \\
\text { intervenção precoce com os medicamentos, que podem ajudar a diminuir a carga } \\
\text { do cuidador, melhorando assim sua qualidade de vida. }\end{array}$ \\
\hline PubMed & $\begin{array}{l}\text { Investigating what } \\
\text { works to support } \\
\text { family carers of } \\
\text { peoplerrath } \\
\text { dementia: a rapid } \\
\text { realist review. }\end{array}$ & $\begin{array}{l}\text { Parkinson } \\
\mathrm{M} \text {, et al. }\end{array}$ & $\begin{array}{c}\text { J Public Health } \\
\text { (Oxf). } 2017 \text { Dec; } \\
\text { 39(4): e290- } \\
\text { e301. }\end{array}$ & $\begin{array}{l}\text { Investiga 'o que funciona } \\
\text { para apoiar os cuidadores } \\
\text { familiares de pessoas com } \\
\text { doença de Alzheimer. }\end{array}$ & $\begin{array}{l}\text { Revisão realista rápida. A seleção e avaliação dos documentos identificaram } \\
\text { cinco temas que abrangem diferentes áreas de apoio ao cuidador familiar: } \\
\text { ampliação dos ativos sociais, fortalecimento dos principais recursos psicológicos, } \\
\text { manutenção do status de saúde física, salvaguarda da qualidade de vida e } \\
\text { garantia da disponibilidade oportuna dos principais recursos externos. Supõe-se } \\
\text { que esses cinco fatores se combinem e interajam para fornecer suporte } \\
\text { biopsicossocial e de serviço crítico, que reforce a 'resiliência' do cuidador e apóie } \\
\text { a manutenção e o sustento dos cuidados familiares. Conclui que o fortalecimento } \\
\text { da resiliência é central para o que funciona para apoiar os cuidadores familiares } \\
\text { de pessoas com demência. }\end{array}$ \\
\hline
\end{tabular}

REAEnf/EJNC | Vol. 2 | e2353 | DOI: https://doi.org/10.25248/REAenf.e2353.2020 Página 6 de 10 


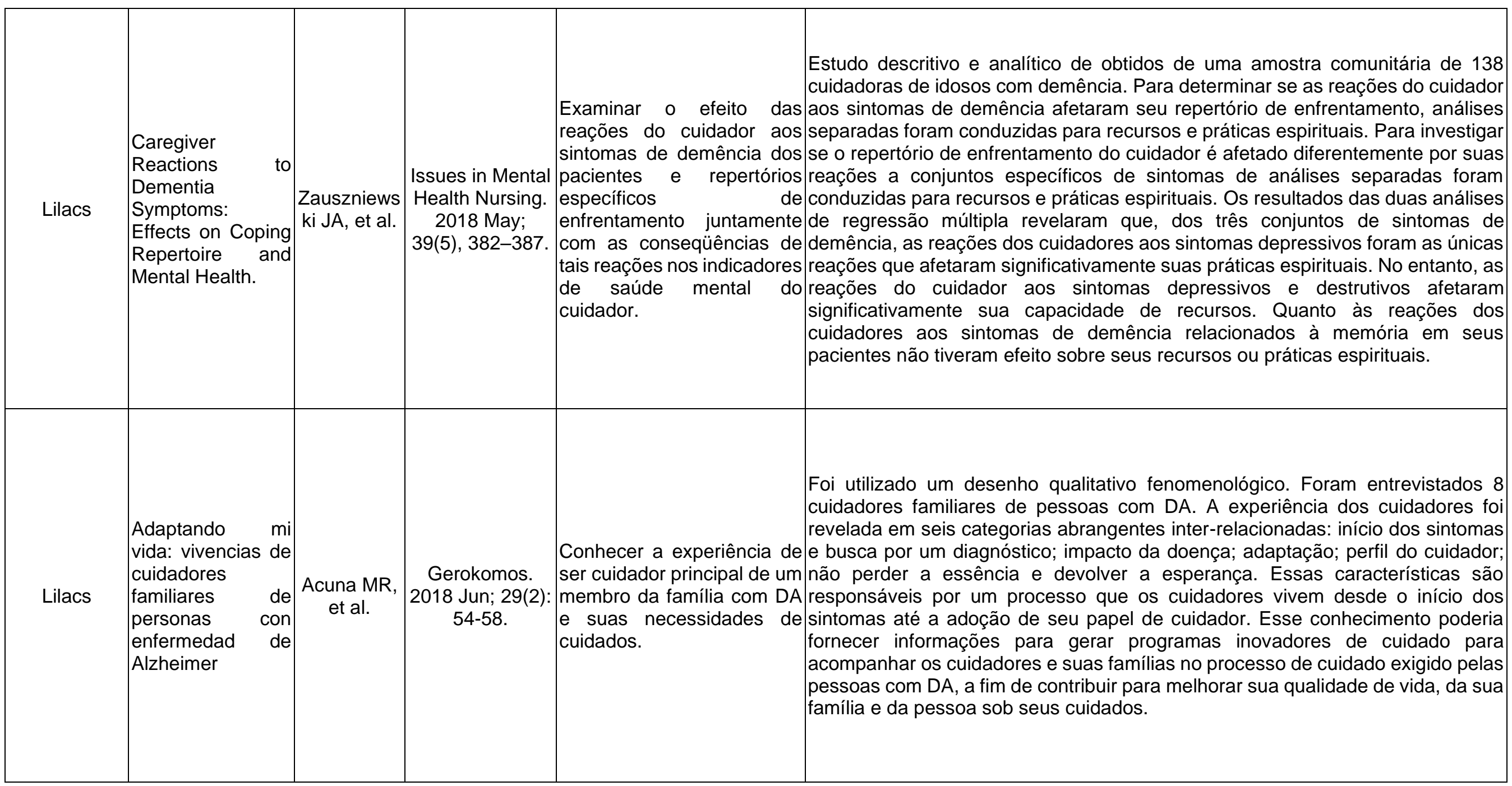

Fonte: Silva PDM, et al., 2019.

REAEnf/EJNC | Vol. 2 | e2353 | DOI: https://doi.org/10.25248/REAenf.e2353.2020 Página 7 de 10 


\section{DISCUSSÃO}

Estudos analisados demonstram que a carga do cuidador a frente de um paciente com DA é considerada de alto risco para sua saúde, e com o passar do tempo, o peso do cuidado vai aumentando, juntamente com as preocupações e uma sobrecarga que tende a aumentar com a evolução progressiva da doença (BERRY B, et al, 2015; GRANO C, et al., 2017; ACUNA MR et al., 2018;).

Um estudo longitudinal realizado na Itália, que avaliou a carga do cuidador a auto-eficácia do cuidador e sintomas depressivos em população de 170 cuidadores de pacientes com DA, demonstrou que carga no momento da primeira avaliação influenciou significativamente a depressão um ano depois e a relação entre a carga na avaliação inicial e os sintomas depressivos um ano depois foi parcialmente mediada pela autoeficácia no controle de pensamentos perturbadores, concluindo que durante um período considerável de tempo, os efeitos da carga do cuidador sobre os sintomas depressivos podem ser explicados pelas crenças de eficácia dos cuidadores no controle de pensamentos perturbadores relacionados às tarefas de cuidar e que intervenções para cuidadores de pacientes com DA podem ajudá-los a enfrentar pensamentos negativos sobre o papel de cuidador (GRANO C, et al., 2017).

Achados semelhantes foram encontrados nos Estados Unidos, onde um estudo transversal investigou como os membros da família enfrentam e gerenciam o declínio funcional do familiar com mostrou que, inicialmente, os indivíduos afetados pela DA eram percebidos como conscientes de seu declínio e recorriam rotineiramente aos membros da família em busca de apoio. Em fases de avanço da doença, onde o déficit de consciência do indivíduo havia diminuído, correspondendo a maior risco ao indivíduo com DA, observou-se perda de confiança na capacidade do indivíduo de regular suas próprias atividades para evitar esses riscos, e os familiares adotaram práticas unilaterais para gerenciar a autonomia do indivíduo em torno dos envolvimentos de suas atividades. Essas práticas geralmente envolviam vários enganos e artifícios para desencorajar os idosos de se envolverem em atividades consideradas potencialmente perigosas. Conclui que o contexto social do trabalho interpretativo em torno da consciência e do risco desempenha um papel importante na maneira como as famílias percebem a capacidade funcional de um idoso e gerenciam o envolvimento de suas atividades (BERRY B, et al, 2015).

No Chile foi observado em pesquisa qualitativa que, em relação ao impacto da doença sobre os cuidadores, os aspectos que mais se destacam foram os econômicos e os relacionados ao enfraquecimento das redes de apoio social. A sobrecarga de cuidado no sentimento de um plano de vida frustrado, onde a vida é percebida apenas no aqui e agora sem ter a capacidade de olhar para o futuro e retomar a vida incorporando esse novo cenário. A maior parte dos cuidadores expressou a sensação de estarem sobrecarregados, o que está relacionado ao grande fardo de ter um parente com DA. Percebeu-se uma exaustão crescente, sem possibilidades reais e contínuas de descanso. Em muitas ocasiões, os membros da família reagiram negativamente de maneira compensatória, com respostas verbais violentas ou inapropriadas a outras pessoas da família (ACUNA MR et al., 2018).

Outro estudo realizado nos Estados Unidos revelou que os sintomas depressivos expressos pelo membro da família com demência tiveram o maior impacto sobre os cuidadores, sendo encontradas associações entre as reações dos cuidadores aos sintomas depressivos expressas pelos familiares com demência e seu repertório de enfrentamento e saúde mental, ou seja, como os cuidadores relataram sentir-se mais perturbados quando os familiares com demência pareciam tristes, chorosos ou preocupados, ou expressavam sentirem-se inúteis, sem esperança ou solitários, ou quando conversavam sobre querer se machucar ou morrer. Relataram também usar menos estratégias de enfrentamento, incluindo menos habilidades de recursos e práticas espirituais e problemas de saúde mental, indicados por maior estresse e sintomas depressivos mais frequentes. Com relação às reações aos sintomas destrutivos de demência expressos pelos membros da família com demência, como argumentatividade, irritabilidade, agressão ou ameaças verbais, volume ou destrutividade, também foram fortemente relacionadas à menor capacidade de recursos, maior estresse e sintomas depressivos mais frequentes nos cuidadores (ZAUSZNIEWSKI JA, et al., 2018). 
Observa-se que as o processo de cuidar de familiares com DA, promove inúmeras mudanças, tanto na vida do paciente quanto na vida do cuidador diariamente, e a pressão psicológica sofrida a cada dia pode resultar em quadros depressivos e transtornos psicológicos, diminuição da qualidade de vida que podem levar o cuidador a sofrer problemas com sua saúde. Verifica-se que estratégias de adaptação aos estressores são fundamentais para o enfrentamento dos cuidadores durante esse processo.

Estudo qualitativo realizado na Espanha, com o objetivo de identificar e analisar os problemas que surgem no processo de adaptação do cuidador às mudanças na assistência à família para uma pessoa afetada pela demência demonstrou que o desempenho do cuidador na sua função é caracterizado por mudanças pessoais, mudanças na pessoa cuidada e mudanças no contexto social e cultural da família e que o desafio de adaptação é o equilíbrio entre os problemas e as formas de enfrentamento. Revela ainda que a falta de apoio formal e o atraso no diagnóstico de demência dificultam essa adaptação, e que esse processo poderia ser melhorado pelo reforço do apoio formal nos estágios iniciais de cuidados reduzindo os estressores do processo de auto-aprendizagem dos cuidadores familiares e adaptando as intervenções para cada fase da evolução da doença (MORENO-CÁMARA S et al., 2016)

Um estudo feito em Xangai na China com objetivo de identificar estressores de cuidado e estratégias de enfrentamento em uma amostra de 18 cuidadores familiares de pessoas com demência concluiu que além dos estressores diretamente relacionados às demandas de cuidar como: sobrecarga devido às limitações do cuidador nas funções físicas, cognitivas ou comportamentais, bem como tensões de papéis, conflitos familiares e pressão do ambiente social. No entanto, os cuidadores demonstraram resiliência utilizando seus recursos de enfrentamento de várias fontes, incluindo experiência pessoal, família, tecnologia da informação, religião e apoio governamental (SUN F, 2013).

Um estudo transversal quantitativo realizado na China para avaliar a carga do cuidador e fatores que a influenciam essa carga entre cuidadores e pacientes com DA, evidenciou que sonolência foi um problema físico comum para os cuidadores, bem como depressão e ansiedade moderada a grave. Um menor estado funcional do paciente foi associado à maior sobrecarga do cuidador. O pior estado físico em comparação com o início do cuidado, menor satisfação com a vida e graus mais altos de depressão e ansiedade foram associados à maior carga do cuidador. Concluindo que problemas de depressão, ansiedade e sono são os principais desafios enfrentados pelos cuidadores familiares de pacientes com doença de Alzheimer. $O$ estado funcional dos cuidadores, menor satisfação com a vida, depressão e ansiedade influenciaram a carga do cuidador. A sobrecarga do cuidador estava relacionada à gravidade da demência do paciente e aos fatores pessoais dos cuidadores (LIU S, et al., 2017).

Em uma recente revisão que objetivou investigar os principais fatores relacionados ao que apoia os cuidadores familiares de pessoas com DA e como esse fatores de apoio podem ser mais amplamente promovidos a fim de beneficiar todos os cuidadores de pessoas com DA, foi observado que a ampliação dos ativos sociais, o fortalecimento dos recursos psicológicos, a manutenção de saúde física, salvaguarda a qualidade de vida e garantia da disponibilidade oportuna dos principais recursos externos. Supõe que esses cinco fatores se combinem e interajam para fornecer suporte biopsicossocial e de serviço crítico, que reforce a resiliência do cuidador e apoie a manutenção e o sustento dos cuidados, concluindo que o fortalecimento da resiliência é o eixo central de apoio dos cuidadores familiares de pessoas com deficiência (PARKINSON $M$ et al., 2017).

\section{CONCLUSÃO}

Os achados desse estudo demonstram alta sobrecarga de estresse e dificuldades de enfrentamento, observados nos cuidadores de familiares com DA. Declínio na vida social, no lazer, no auto-cuidado, no tempo dedicado a sua vida particular, geram impactos negativos, e com passar do tempo traz problemas à sua saúde, sendo imprescindível uma atenção voltada ao cuidador, a sua saúde, a fim de evitar o desenvolvimento e o surgimento de doenças que podem afetar sua vida e trazer prejuízos que podem ser até mesmo irreversíveis. 


\section{REFERÊNCIAS}

1. ACUNA MR, et al. Adapting my life: experiences of caregivers of people with Alzheimer's disease. Gerokomos (Madr., Ed. impr.), 2018; 29(2): 54-58.

2. BERRY B, et al. How family members manage risk around functional decline: The autonomy management process in households facing dementia. Social Science \& Medicine, 2015; 130: 107-114.

3. CHEN CT, et al. Neuropsychiatric symptoms in Alzheimer's disease: associations with caregiver burden and treatment outcomes. QJM: An International Journal of Medicine, 2017; 110(9): 565-570.

4. GRANO C, et al. The relationship between caregiving self-efficacy and depressive symptoms in family caregivers of patients with Alzheimer disease: a longitudinal study. International Psychogeriatrics, 2017; 29(7): 1095-1103.

5. JING W, et al. Factors influencing quality of life of elderly people with dementia and care implications: A systematic review. Archives of Gerontology and Geriatrics, 2016; 66: 23-41.

6. LIU S, et al. Caregiver burden and prevalence of depression, anxiety and sleep disturbances in Alzheimer's disease caregivers in China. Journal of Clinical Nursing, 2017; 26(9-10): 1291-1300.

7. LUCHESI BM, et al. Evaluation of depressive symptoms in older caregivers. Arch Clin Psychiatry, 2015; 42(2): 4551.

8. MONTEIRO AMF, et al. Coping strategies among caregivers of people with Alzheimer disease: a systematic review. Trends in Psychiatry and Psychotherapy, 2018; 40(3): 258-268.

9. MOON H, DILWORTH-ANDERSON P. Baby boomer caregiver and dementia caregiving: findings from the National Study of Caregiving. Age Ageing, 2015; 44(2): 300-306.

10. MORENO-CÁMARA S, et al. Problemas en el proceso de adaptación a los cambios en personas cuidadoras familiares de mayores con demencia. Gaceta Sanitaria, 2016; 30(3): 201-207.

11. PARKER G, et al. Meta-revisão de Evidências Internacionais sobre Intervenções para Apoiar Cuidadores . Universidade de York, York: Unidade de Pesquisa em Políticas Sociais, 2010. 85 p.

12. PARKINSON M, et al. Investigating what works to support family carers of people with dementia: a rapid realist review. Journal of Public Health, 2017; 39(4): 290-301.

13. SILVA MVF, et al. Alzheimer's disease: risk factors and potentially protective measures. Journal of Biomedical Science, 2019; 26(33).

14. SUN F. Caregiving stress and coping: A thematic analysis of Chinese family caregivers of persons with dementia. Dementia, 2014; 13(6), 803-818.

15. URSI ES, GAVÃO CM. Prevenção de lesões de pele no perioperatório: revisão integrativa da literatura. Rev LatinoAm Enfermagem. 2006; 14(1): 124-31.

16. WORLD HEALTH ORGANIZATION. Dementia: a public health priority [Internet]. Geneva:WHO;2012

17. ZAUSZNIEWSKI JA, et al. Caregiver Reactions to Dementia Symptoms: Effects on Coping Repertoire and Mental Health. Issues in Mental Health Nursing, 2018; 39(5), 382-387. 